\section{Accuracy of tablet counts estimated by members of the public and healthcare professionals}

\author{
Hyun-Sik Choi, Yoon Hee Choi \\ Department of Emergency Medicine, Ewha Womans University School of Medicine, Seoul, Korea
}

Objective Intentional and accidental drug intoxication is commonly seen in the emergency department. When treating intoxicated patients, accessing the amount of the ingested drug is crucial albeit often difficult. We investigated the accuracy of estimating tablet counts when participants were asked to hold tablets in their fists and hands (semi-quantitative terms).

Methods The widths and lengths of the participants' hands were measured. Then, the subjects were asked to hold 5-mm round, 10-mm round, 10- $\mathrm{mm}$ oval, and 15-mm elliptical tablets using their hands and fists and to estimate the number of tablets they were holding. Differences between the estimated and actual numbers of tablets were examined.

Results A total of 47 members of the public and 32 healthcare professionals were included in our study. In our analyses of the differences between the actual and estimated amounts of tablets held in the participants' hands and fists, we found that the actual amount was higher than the estimated amount for all tablet types and in both groups. When participants held the tablets in the same manner (handful or fistful), the differences between the actual and estimated amounts were greater for 5- than 15-mm-sized tablets $(\mathrm{P}<0.05)$.

Conclusion The treatment of patients presenting with drug overdoses to the emergency department should be based on the assumption that the actual amount of drugs the patients ingested is likely greater than the amount the patients state.

Keywords Drug overdose; Emergency service, hospital; Knowledge

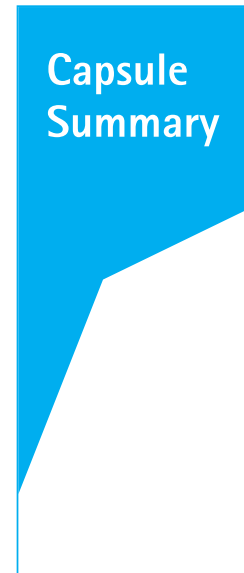

What is already known

Drug intoxication is one of the common causes of emergency department visits. However, patients visiting the emergency department due to intoxication rarely know the accurate amount of the toxic substance they ingested. A previous study investigated the accuracy of the volume of toxic liquid ingestions in adults. However, there is no investigation on the accuracy of the amounts of ingested tablets.

\section{What is new in the current study}

The author investigated the estimated and actual tablet counts when members of the public and healthcare professionals were asked to hold tablets in their fists and hands. The actual amount was higher than the estimated amount for all forms of tablets.
eISSN: 2383-4625

Received: 26 December 2014

Revised: 13 March 2015

Accepted: 16 March 2015

Correspondence to: Yoon Hee Choi Department of Emergency Medicine, Ewha Womans University School of Medicine, 1071 Anyangcheon-ro, Yangcheon-gu, Seoul 158-710, Korea E-mail: like-lemontea@hanmail.net

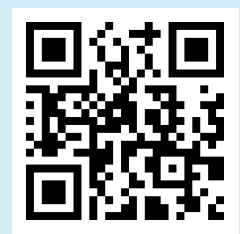

How to cite this article:

Choi HS, Choi YH. Accuracy of tablet counts estimated by members of the public and healthcare professionals. Clin Exp Emerg Med 2015;2(3):168-173.

This is an Open Access article distributed under the terms of the Creative Commons Attribution Non-Commercial License (http:// creativecommons.org/licenses/by-nc/3.0/). 


\section{INTRODUCTION}

Drug intoxication is one of the common causes of emergency department (ED) visits. Intoxication can be either intentional or accidental. In adults, intoxication occurs through excessive ingestion of toxic substances with suicidal intent or by accidental poisoning. In contrast, intoxications in children are mostly accidental. Intoxicated patients who visit the ED make up about 1\% of all ED patients.'

The proportion of deaths caused by intoxication is relatively small (0.5\% to $1.4 \%$ of all deaths). ${ }^{1}$ Korea has been reported as having the highest suicide rate among all OECD (Organization for Economic Cooperation and Development) countries in 2011, and its suicide rate has been increasing. ${ }^{2}$ The substances associated with intoxications vary greatly and include tablets or liquid drugs, agricultural chemicals, industrial agents, acids, and alkaline substances. It is crucial to know the amount of the toxic substances patients have ingested to identify adequate treatment strategies. However, patients visiting the ED due to intoxication rarely know the accurate amount of the toxic substance they ingested. Moreover, if patients are unconscious, they are unable to answer the physician's questions. Hence, the amount of ingested poisonous material is often calculated using either witness or caregiver statements, or is estimated by physicians. In these cases, the amount is typically described using semi-quantitative terms. For prescription drugs, the maximum number a patient might have ingested can be estimated based on prescriptions or the amount of leftover drugs. In contrast, the amounts of ingested drugs that are sold in bulk containers such as acetaminophen and ibuprofen, hypnotics, sedatives, or antipsychotics are typically described by patients and caregivers using the semi-quantitative expressions 'fistful' or 'handful.' Even when patients presenting with drug intoxication have stable vital signs and are alert, complications such as rhabdomyolysis and acute renal failure can occur after ingestion of the sedative doxylamine, and hepatic failure can occur after ingestion of acetaminophen. Hence, estimating the amount of the drug that the patient has ingested is helpful for treatment. However, no investigation on the accuracy of the reported amounts of ingested drugs (described as handful or fistful, terms that are often used to describe the amount of drugs a patient has ingested) has been conducted yet. Moreover, there are no data available on how patients and healthcare professionals estimate the amounts of handful or fistful, and if their estimations are accurate.

We investigated the estimated and actual tablet counts when laypersons and healthcare professionals were asked to hold tablets in their fists and hands (semi-quantitative terms) and determined the accuracy of their estimations.

\section{METHODS}

\section{Study population}

The study participants were healthy adults without handgrip disorders who were recruited from a university hospital to participate in our study that lasted 15 days (from January 8 to January 22 , 2014). All participants understood the purpose of the study and provided informed consent. The participants were selected from 2 populations-members of the non-medical personnel and healthcare professionals. This study was conducted in agreement with the Declaration of Helsinki, and the institutional review board of Ewha Womans University Mokdong Hospital approved the study.

\section{Study protocol}

Measurement of the participants' hand sizes

The hand surface areas of the participants were measured based on the maximum width of the palm and the length from the bottom of the palm to the tip of the longest finger. To present the hand size, a 2-dimensional method that multiplied the width and length of the hand was used (Fig. 1).

\section{Tablet types}

We used 4 different types of tablets in this study: $5-\mathrm{mm}$ round tablets (doxylamine succinate 25 mg; Aron, Korea Arlico Pharm, Jincheon, Korea), 10-mm round tablets (acetylsalicylic acid 500 mg; Aspirin, Bayer Korea, Seoul, Korea), 10-mm oval tablets (alprazolam 0.25 mg; Xanax, Pfizer Pharmaceuticals, Barceloneta, Spain), and 15-mm elliptical tablets (acetaminophen $500 \mathrm{mg}$; Tylenol, Janssen Korea, Hwaseong, Korea).

\section{Measurements}

The participants were instructed to hold as many tablets as pos-

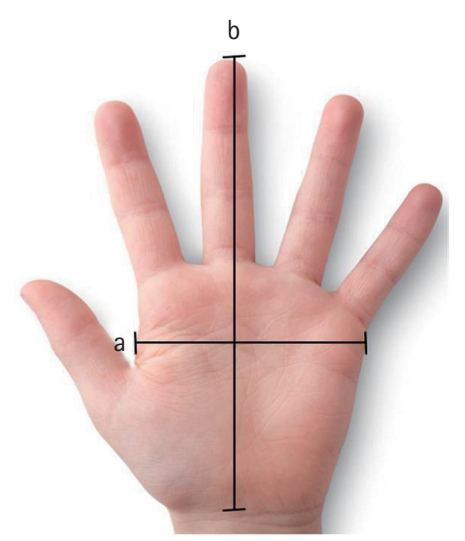

Fig. 1. Measurement of hand size. (a) Hand width and (b) length from the lower margin of the palm to the top of the longest finger. 
sible of each type in their fists and then their hands. In both cases, the number of tablets was not visible to the individuals (Fig. 2). They then were told to put the tablets on a table without looking at them and to estimate the number of tablets they had held in their firsts or hands. These estimates were recorded. Last, the actual number of the tablets was counted; however, these numbers were not disclosed to the participants.
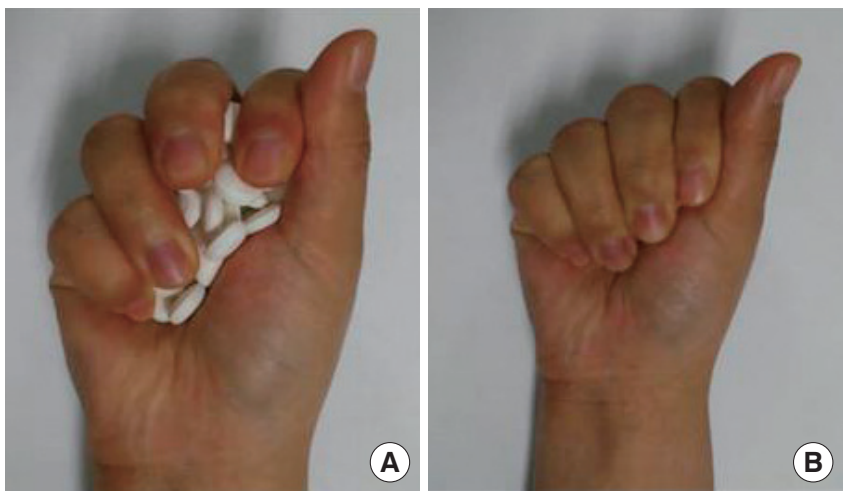

Fig. 2. Manner of holding. (A) Handful and (B) fistful.

Table 1. Characteristics of healthcare professionals and members of the public

\begin{tabular}{lccc}
\hline Characteristics & $\begin{array}{c}\text { Health care } \\
\text { professional }\end{array}$ & Layperson & P-value \\
\hline Age $(\mathrm{yr})$ & $26.3 \pm 3.1$ & $25.3 \pm 2.3$ & 0.327 \\
Male $(\%)$ & $8(25.0)$ & $2(4.3)$ & 0.057 \\
Hand size $(\mathrm{mm})$ & & & \\
$\quad$ Width & $76.7 \pm 9.0$ & $76.1 \pm 9.7$ & 0.438 \\
Length $^{\text {a) }}$ & $173.2 \pm 11.8$ & $173.6 \pm 12.6$ & 0.214 \\
\hline
\end{tabular}

Values are presented as mean \pm SD or number $(\%)$.

${ }^{\text {a) }}$ Measured from the bottom of the palm to the tip of the longest finger.

\section{Data analysis}

Cross-tabulation was performed for non-continuous variables using PASW ver. 18.0 (SPSS Inc., Chicago, IL, USA), and the Student's t-test was used for continuous variables. Analysis of variance with Bonferroni correction was used where appropriate. A P-value $<0.05$ was considered statistically significant.

\section{RESULTS}

A total of 47 members of the public ( 2 men and 45 women; mean age, $25.39 \pm 2.30$ years) and 32 healthcare professionals ( 8 men and 24 women; mean age, $26.31 \pm 3.15$ years) were included in this study. The mean palm widths of the members of the public and healthcare professional were $76.1 \pm 9.70$ and $76.6 \pm 9.01 \mathrm{~mm}$, respectively. We found mean hand lengths of $173.56 \pm 12.63$ and $173.19 \pm 11.83 \mathrm{~mm}$ for the members of the public and healthcare professionals, respectively. We did not detect statistically significant differences in the participants' demographic characteristics (Table 1) and the actual amount of tablets for both handful and fistful $(P>0.05)$ (Table 2).

When comparing the differences between the actual and estimated amounts of tablets, the actual amount was higher than the estimated amount for all forms of tablets in both groups for both handful and fistful (Table 2). When the participants held the tablets in the same manner (using their hands or fists), the differences between the actual and estimated amounts were greater for the 5-mm-sized than the 15-mm-sized tablets $(P<0.05)$. When we compared healthcare professionals and non-medical personnel, we found significant differences in $10-\mathrm{mm}$ round tablet for handful and fistful $(P<0.05)$. The estimated tablet amounts reported by the healthcare professionals were lower than those reported by the non-medical personnel (Figs. 3, 4).

Table 2. Estimated and actual tablet count and the difference between the two by tablet type and manner of holding

\begin{tabular}{|c|c|c|c|c|c|c|c|c|c|}
\hline & \multicolumn{4}{|c|}{ Health care provider } & \multicolumn{4}{|c|}{ Layperson } & \multirow{2}{*}{$\begin{array}{l}\text { P-value for } \\
\text { difference }\end{array}$} \\
\hline & Estimated count & Actual count & Difference $^{a)}$ & P-value & Estimated count & Actual count & Difference $^{a)}$ & P-value & \\
\hline \multicolumn{10}{|c|}{ 5-mm round tablet } \\
\hline Handful & $85.8 \pm 45.1$ & $165.2 \pm 40.6$ & $79.4 \pm 47.9$ & 0.033 & $76.5 \pm 25.8$ & $137.7 \pm 41.3$ & $61.3 \pm 47.7$ & 0.043 & 0.088 \\
\hline Fistful & $45.6 \pm 23.0$ & $75.3 \pm 31.9$ & $29.7 \pm 29.5$ & 0.008 & $44.6 \pm 17.0$ & $70.0 \pm 22.4$ & $25.4 \pm 19.0$ & 0.000 & 0.468 \\
\hline \multicolumn{10}{|c|}{$10-\mathrm{mm}$ round tablet } \\
\hline Handful & $41.8 \pm 15.7$ & $52.4 \pm 16.3$ & $10.7 \pm 14.4$ & 0.000 & $37.8 \pm 12.2$ & $41.3 \pm 11.0$ & $3.6 \pm 11.2$ & 0.000 & 0.016 \\
\hline Fistful & $22.8 \pm 7.8$ & $30.3 \pm 13.5$ & $7.6 \pm 9.8$ & 0.000 & $21.2 \pm 6.5$ & $22.6 \pm 6.4$ & $1.4 \pm 6.7$ & 0.001 & 0.003 \\
\hline \multicolumn{10}{|c|}{ 10-mm oval tablet } \\
\hline Handful & $70.9 \pm 37.6$ & $123.0 \pm 39.3$ & $52.0 \pm 47.0$ & 0.033 & $66.3 \pm 19.9$ & $104.0 \pm 25.0$ & $37.7 \pm 24.5$ & 0.003 & 0.122 \\
\hline Fistful & $41.3 \pm 23.3$ & $68.5 \pm 34.0$ & $27.3 \pm 23.9$ & 0.000 & $37.0 \pm 11.7$ & $57.3 \pm 15.4$ & $20.3 \pm 15.4$ & 0.009 & 0.153 \\
\hline \multicolumn{10}{|c|}{ 15-mm elliptical tablet } \\
\hline Handful & $33.3 \pm 9.5$ & $38.6 \pm 11.6$ & $5.3 \pm 12.0$ & 0.039 & $29.4 \pm 7.8$ & $29.9 \pm 8.1$ & $0.6 \pm 6.6$ & 0.000 & 0.046 \\
\hline Fistful & $18.7 \pm 6.6$ & $21.9 \pm 9.6$ & $3.2 \pm 8.5$ & 0.003 & $16.7 \pm 5.9$ & $17.0 \pm 5.2$ & $0.3 \pm 5.6$ & 0.000 & 0.098 \\
\hline
\end{tabular}

a)The differences between the estimated and actual counts were calculated by subtracting the estimated from the actual counts. 


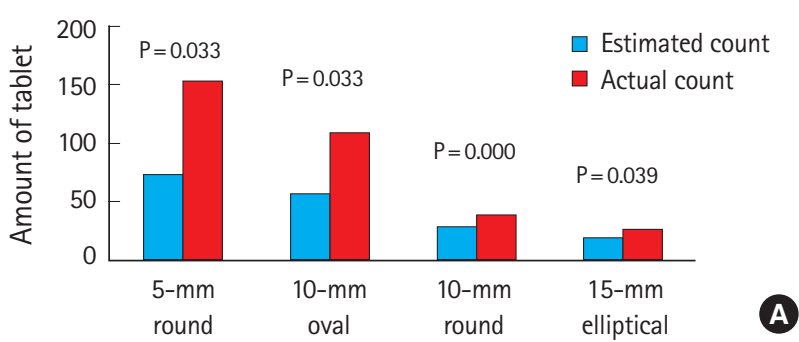

Fig. 3. Handful grip of (A) healthcare professionals and (B) layperson.

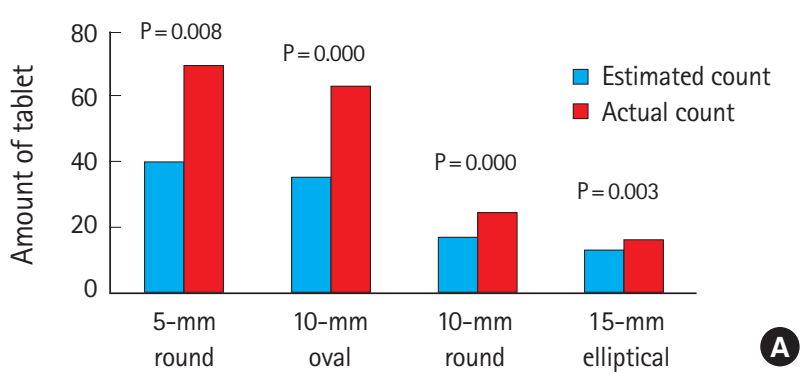

Fig. 4. Fistful grip of (A) healthcare professionals and (B) layperson.

\section{DISCUSSION}

Drug intoxication is one of the most common causes of ED visits. According to an ED-based injury surveillance in Korea, the number of deaths by intoxication was 1,787 in 2010. Of these, $31.3 \%$ were accidental and 59.3\% suicidal intoxications; it could not be determined if the remaining 9.4\% were intentional or accidental. ${ }^{2}$ In the US, 31,759 of $41,592(76 \%)$ individuals who died of intoxication in 2009 presented with unintentional intoxication, and among patients with unintentional intoxication, a drug overdose was the cause of death in $91 \%$ of patients. ${ }^{3}$ In the US, intoxications are often asymptomatic and non-fatal, whereas the percentage of patients experiencing life threatening intoxication is very high in Korea. ${ }^{2,3}$ An ED-based intoxication survey conducted in Korea in 2009 showed that the admission and mortality rates of intoxicated patients were $29.55 \%$ and $4.4 \%$, respectively. ${ }^{2}$ Knowing the amount of poisonous material or drugs that patients ingested is very important as evaluations of drugs' and poisons' toxicities and predictions of patient survival are based on these amounts. ${ }^{4}$

In Korea, everybody can purchase certain drugs in bulk, as overthe-counter drugs that do not require a prescription can be sold at convenience stores. ${ }^{5}$ The types of drugs that can be purchased without prescriptions are mostly antipyretic analgesics such as Tylenol and Advil syrup, decongestants, and some hypnotics such as doxylamines. Drugs that do not require a prescription by a physician or medication counseling are often perceived as not harm-
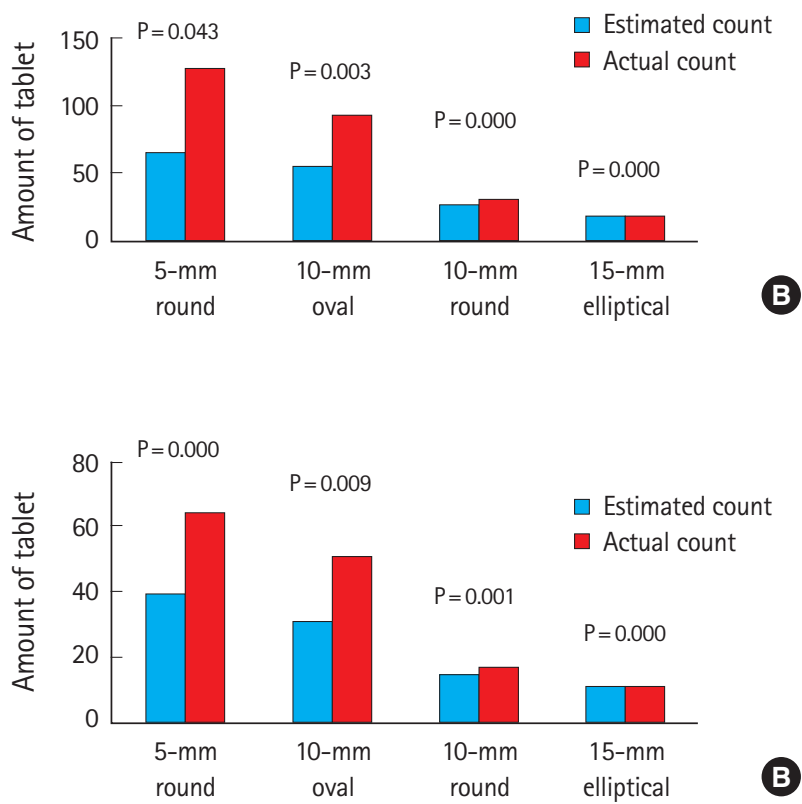

ful. If individuals of various age groups can acquire a large amount of drugs easily, drug intoxication is a significant risk. When patients take drugs that are sold in the form of a container for bulk storage, or when they save up their drugs and take them all at once, they typically describe the amount of drugs they took using the expressions 'handful' and 'fistful.' Doxylamine and acetaminophen overdoses are often seen in the ED, and the risks of these drugs have been demonstrated. ${ }^{6,7}$ Rhabdomyolysis is one of the serious complications of a doxylamine overdose. It occurred in $21 \%$ to $59 \%$ in doxylamine overdose, and 19\% of patients with rhabdomyolysis developed acute renal failure. ${ }^{6,7}$

Estimations of volumes and dimensions have been conducted in other settings, including those in relation to blood loss, body size, and tissue volumes. These studies have been found to be error-prone, and underestimation has been particularly pointed out. . $^{-11}$ An investigation of the amount of tablets measured in 'handful' and 'fistful', terms that are typically used to express the amount of drug ingestion, has not been performed yet. In this study, we therefore assessed the predicted and actual tablet counts when healthcare professionals and members of the public were asked to hold tablets in their fists and hands (semi-quantitative terms). We then calculated the differences between the predicted and actual counts.

In our study, we used tablets of sizes that are typically used by patients: 5, 10, and $15 \mathrm{~mm}$. When the participants held a fistful of 5-mm-sized tablets, they could hold 75.34 231.90 (healthcare professionals) and $69.96 \pm 22.36$ (members of the public) tablets. 
For a handful of 5-mm-sized tablets, we found counts of $165.16 \pm$ 40.55 (healthcare professionals) and 137.74 441.32 (members of the public). When the participants were asked to hold a fistful and handful of $10-\mathrm{mm}$-sized round tablets, healthcare professionals could hold $30.31 \pm 13.51$ and $52.41 \pm 16.28$, respectively, and members of the public could hold $22.55 \pm 6.37$ and $41.32 \pm 10.95$ tablets, respectively. For a fistful and handful of 10-mm-sized oval drugs, we observed tablet counts of $68.50 \pm 34.00$ and $122.97 \pm$ 39.34 , respectively, among healthcare professionals and $57.34 \pm$ 15.38 and $103.98 \pm 25.03$, respectively, among members of the public. Last, when asked to hold a fistful and handful of 15-mmsized tablets, healthcare professionals could hold $21.88 \pm 9.63$ and $38.63 \pm 11.56$, respectively, and members of the public could hold $16.96 \pm 5.16$ and $29.94 \pm 8.14$, respectively. In this study, we found that the actual amount of drugs was higher than the estimated amount among both healthcare professionals and members of the public. This finding was irrespective of the manner of holding (handful vs. fistful). The differences between the actual and estimated amounts increased as the size of the tablets decreased. Therefore, the actual amount of drug ingestion might be greater than the amount that patients or caregivers describe when patients visit the ED.

It was found that when individuals held a handful of tablets, they were able to hold double the amount of tablets than when they held a fistful of tablets. This indicates that even when patients have hands of the similar size, the number of tablets they can hold in their hands can differ significantly depending on the manner of holding the tablets. Therefore, including the method patients use when holding tablets is required when patients visit the ED.

In our study, healthcare professionals showed a tendency to underestimate the tablet count. Physicians' propensity of underestimating blood loss has been reported in other studies across a range of medical and surgical specialties..$^{811,12}$ However, we are not suggesting that physicians should simply ignore the estimates of tablet amount from patients and caregivers.

Our study has several limitations. First, the size of the tablets was solely based on their diameter, and the volume and shape of the tablets were not considered. If same-diamerter tablets have different volumes and shapes, the number of tablets individuals can hold in their hands might be different. In this study, even when the same diamerter 10-mm-sized tablets were used, our findings were different based on the tablets' shapes (round vs. oval). Therefore, additional studies considering the tablets' volumes are needed. Second, the education level of the study participants could have affected the estimated tablet counts. However, all members of the public included in this study were university graduates. Fu- ture studies should be conducted in various population clusters.

The treatment of patients presenting with drug overdoses to the ED should be based on the assumption that the actual amount of drugs the patients ingested is likely greater than the amount the patients state. Furthermore, it must be considered that the difference between the estimated and actual tablet counts might be even greater for small-sized tablets, requiring anticipation of a fatal situation.

\section{CONFLICT OF INTEREST}

No potential conflict of interest relevant to this article was reported.

\section{REFERENCES}

1. Korean Statistical Information Service. Cause of death. Deaths by cause(103 item)/by sex [Internet]. Daejeon: Statistics Korea; 2015 [cited 2015 May 21]. Available from: http://kosis.kr/ statHtml/statHtml.do?orgld = 101\&ttblld=DT_1B34E02\&tvw_ $c d=$ MT_ZTITLECtlist_id $=$ D11\&tsegNo $=$ Etlang_mode $=$ koctlan guage $=$ korEtobj_var_id $=$ ctitm_id $=$ ctconn_path $=$ E1\# .

2. Korean Statistical Information Service. Mortality rate by cause (per 100,000 population)(OECD) [Internet]. Daejeon: Statistics Korea; 2014 [cited 2015 May 21]. Available from: http://kosis. $\mathrm{kr} / \mathrm{statHtml} / \mathrm{statHtml}$.do?orgld = 101\&tblld = DT_2KAAC13 OECDEtvw_cd $=$ MT_RTITLECtlist_id = UTIT_OECD_LCtseqNo = Etlang_mode $=$ koctlanguage $=$ korEtobj_var_id $=$ Ctitm_id $=$ Etconn_path = A4\#.

3. Bronstein AC, Spyker DA, Cantilena LR Jr, Green JL, Rumack BH, Giffin SL. 2009 Annual report of the American Association of Poison Control Centers' National Poison Data System (NPDS): 27th annual report. Clin Toxicol (Phila) 2010;48:9791178.

4. Hitchings AW, Wood DM, Warren-Gash C, Gil Rivas S, Dargan Pl. Determining the volume of toxic liquid ingestions in adults: accuracy of estimates by healthcare professionals and members of the public. Clin Toxicol (Phila) 2013;51:77-82.

5. Pharmaceutical Affairs Act of 2013, Pub. L. No. 13219 (Mar 13, 2015).

6. Kim HJ, Oh SH, Youn CS, et al. The associative factors of delayed-onset rhabdomyolysis in patients with doxylamine overdose. Am J Emerg Med 2011;29:903-7.

7. Jo Yl, Song JO, Park JH, et al. Risk factors for rhabdomyolysis following doxylamine overdose. Hum Exp Toxicol 2007;26:61721.

8. Yoong W, Karavolos S, Damodaram M, et al. Observer accura- 
cy and reproducibility of visual estimation of blood loss in obstetrics: how accurate and consistent are health-care professionals? Arch Gynecol Obstet 2010;281:207-13.

9. Toledo P, McCarthy RJ, Hewlett BJ, Fitzgerald PC, Wong CA. The accuracy of blood loss estimation after simulated vaginal delivery. Anesth Analg 2007;105:1736-40.

10. Greene S, Dargan P, Shin GY, Jones Al. Doctors and nurses es- timation of the weight of patients: a preventable source of systematic error. J Toxicol Clin Toxicol 2004;42:611-5.

11. Schuld J, Kollmar O, Seidel R, Black C, Schilling MK, Richter S. Estimate or calculate? How surgeons rate volumes and surfaces. Langenbecks Arch Surg 2012;397:763-9.

12. Beer HL, Duvvi S, Webb CJ, Tandon S. Blood loss estimation in epistaxis scenarios. J Laryngol Otol 2005;119:16-8. 